\title{
A Cross-Sectional Study in Patients with Severe COPD to Assess the Perception of Symptom Variability (COPVAR) in the Middle East and Africa
}

This article was published in the following Dove Press journal: International Journal of Chronic Obstructive Pulmonary Disease

Nurdan Kokturk, (D)' Wael Abuharbid, (D) ${ }^{2}$ Amr S Albanna, (D) ${ }^{3}$ Hakan Gunen, ${ }^{4}$ Alev Gurgun, ${ }^{5}$ Mousa Khadadah, $^{6}$ Elmas Malvolti, ${ }^{7}$ Medhat Soliman, (D) ${ }^{8}$ Richard van Zyl-Smit, (D) ${ }^{9}$

Noureddine Zidouni, (1D ${ }^{10}$ Ashraf Alzaabi ${ }^{\prime \prime}$

'Department of Chest Diseases, Faculty of Medicine, Gazi University, Ankara, Turkey; ${ }^{2}$ AstraZeneca, Dubai, United Arab Emirates; ${ }^{3}$ King Abdullah International Medical Research Center, King Saud Bin Abdulaziz University for Health Sciences, Jeddah, Saudi Arabia; ${ }^{4}$ Clinic of Chest Diseases, Sureyyapasa Chest Diseases and Chest Surgery Training and Research Hospital, Istanbul, Turkey; ${ }^{5}$ Department of Chest Diseases, Ege University Faculty of Medicine, Izmir, Turkey; ${ }^{6}$ Department of Medicine, Faculty of Medicine, Kuwait University, Jabriya, Kuwait; ${ }^{7}$ AstraZeneca, Luton, UK; ${ }^{8}$ Kasr El Aini Faculty of Medicine, Cairo University, Cairo, Egypt; ' University of Cape Town Lung Institute, Department of Medicine and Division of Pulmonology, Groote Schuur Hospital, Cape Town, South Africa; ${ }^{10}$ Department of Pulmonology, University Hospital Issad Hassani, Béni Messous, Alger, Algeria;

"'Respiratory Medicine Division, Zayed Military Hospital, Abu Dhabi, UAE

Correspondence: Nurdan Kokturk Department of Pulmonary Medicine, Gazi University School of Medicine, Emniyet Mahallesi, Mevlana Bulvarı, No 29, Yenimahalle, Ankara 06560, Turkey Tel +90312 2026121

Email kokturk.nurdan@gmail.com
Purpose: This study was performed to assess symptom variability and its impact on morning activities in stable patients with severe COPD in the Middle East and Africa (MEA) countries.

Patients and methods: Non-interventional, cross-sectional study (NCT03425760) in patients with severe COPD (GOLD 2015, C, or D categories). Symptom variability was assessed directly by interviewing the patient and using the Global Chest Symptoms Questionnaire (GCSQ). The impact on morning activities was assessed using the Capacity of Daily Living during the Morning (CDLM) and the Morning Activities and Symptoms Questionnaire (MASQ)

Results: A total of 3253 patients (mean \pm SD age: $64.1 \pm 9.5$ years, 90.3\% males) were enrolled. Overall, $81.6 \%$ and $83.4 \%$ of patients reported weekly and daily symptom variability, respectively. The number of exacerbations in the previous year, smoking cessation, and COPD GOLD D were the most consistent factors associated with symptom variability. The GCSQ score was significantly higher $(\mathrm{p}<0.001)$ in GOLD D than in GOLD C patients at each time during the day. In GOLD D, the mean $( \pm \mathrm{SD})$ GCSQ score was higher at night $(1.6 \pm 1.2$, $\mathrm{p}<0.001)$ and in the morning $(1.5 \pm 1.0, \mathrm{p}<0.001)$ than in the afternoon $(1.3 \pm 0.9)$, suggesting daytime variability of breathlessness and chest tightness. Overall, $60.0 \%$ of GOLD D patients (versus $13.6 \%$ GOLD $\mathrm{C}, \mathrm{p}<0.0001$ ) had difficulty getting out of bed due to COPD. Patients with symptom variability had significantly more difficulty to get out of bed, especially patients with chest tightness variability $(\mathrm{p}<0.0001)$ and wheezing variability $(p<0.0001)$. The CDLM global score was significantly lower $(p<0.0001)$ in GOLD D than in GOLD C patients $(3.5 \pm 1.1$ and $4.6 \pm 3.5$, respectively). Daily variability in chest tightness and wheezing was also significantly associated with CDLM scores $(\mathrm{p}<0.0001)$.

Conclusion: In MEA countries, patients with severe stable COPD reported significant daily and weekly symptom variability which affects morning activities, particularly in GOLD D patients.

Keywords: breathlessness, exacerbation, GOLD C, GOLD D, quality of life

\section{Introduction}

According to the World Health Organization, chronic obstructive pulmonary disease (COPD) is the 3 rd leading cause of death worldwide. ${ }^{1}$ As compared to other diseases, this relative increase in mortality is driven by the expansion of the smoking epidemic and reduction in mortality from other common causes of death, such as ischemic heart disease, together with aging of the world population. COPD is the 6th leading cause of disability-adjusted life years (DALYs) in 
middle-income countries, and is expected to become the 5 th leading cause of DALYs worldwide in $2030 .^{2}$

COPD is characterized by persistent airflow limitation associated with multiple respiratory symptoms including dyspnea, cough, sputum production, and wheeze. Anorexia, fatigue, and weight loss may also be present in more severe disease. Although COPD patients may experience flare-ups of symptoms, requiring additional treatment, ${ }^{2}$ COPD is generally considered a stable condition. There is, however, evidence of daily and weekly variability in symptom perception. ${ }^{3}$

In 2015, the Global Initiative for Obstructive Lung Disease (GOLD) recommended classifying disease severity into different risk groups (A-D) based on symptoms, lung function, and exacerbation risk. ${ }^{4}$ Symptoms are assessed by the COPD Assessment Test ${ }^{\mathrm{TM}}$ (CAT) or modified Medical Research Council (mMRC) dyspnea scale. High risk is determined by the degree of airflow limitation using lung function (forced expiratory flow rate in one second (FEV1)) and/or by the number of exacerbations in the previous 12 months. Patients with FEV $1<50 \%$ predicted and/or those with $\geq 2$ past-year exacerbations or more than one exacerbation that leads to hospitalization have a high risk (risk groups C and D). The current 2019 GOLD guidelines have reduced the emphasis on lung function and currently use symptoms and exacerbations to categorize patients. ${ }^{4}$

Adding to exacerbations, patients may experience seasonal, weekly, or even daily worsening of symptoms not consistently fitting an exacerbation episode. The foregoing perceived symptom variability is a factor directly affecting disease impact on daily activities, quality of life (QOL), and prognosis. ${ }^{5}$ In the Multi-Centre Intervention and Characterization Study, perceived symptoms started to increase 2 weeks prior to an exacerbation. ${ }^{6}$ Effective management of stable COPD is thus crucial in alleviating symptoms, preventing exacerbations, and slowing the progression of the disease.

Although COPD symptoms have been extensively reviewed in the literature, daily and weekly variation of symptoms and their impact on daily life activities have received less attention, especially in the Middle East and Africa (MEA) region. No published study in MEA has investigated the fluctuation of COPD symptoms, treatment patterns, and their consistency with GOLD guidelines. This cross-sectional study conducted in the MEA region, therefore, aimed to assess stable GOLD (2015) C and
D COPD patients' perception of daily and weekly symptom variability, and their impact on daily activities.

\section{Patients and Methods}

COPVAR was a multinational, multicenter, noninterventional, cross-sectional observational study performed in 8 MEA countries including Algeria, Egypt, Kuwait, Qatar, Kingdom of Saudi Arabia, Turkey, United Arab Emirates, and South Africa (NCT03425760). The primary objective was to describe the perception of daily (i.e. day-to-day) and weekly variability of symptoms in patients with stable GOLD C and GOLD D COPD according to the 2015 GOLD guidelines, ${ }^{4}$ and their impact on activities of daily living. Comparison between GOLD $\mathrm{C}$ and GOLD D was a secondary study outcome.

Patients enrolled in the study were selected from pulmonary outpatient clinics during the study period (December 2015 to July 2017). Consecutive patients were included if they fulfilled the following criteria: male or female patients over 45 years of age with stable COPD GOLD C/D according to GOLD 2015 guidelines, maintained on COPD treatment, and provided signed informed consent. The diagnosis of COPD was confirmed by spirometry performed in the past 12 months with $\mathrm{FEV}_{1} / \mathrm{FVC}$ $<0.7$ and $\mathrm{FEV}_{1}<50 \%$ predicted. Patients were excluded if they had recently or were currently exacerbating (defined as worsening of COPD symptoms leading to treatment with antibiotics and/or a short course of oral steroids and/or hospitalization or emergency room visit in the previous 3 months), any other chronic respiratory disease was present such as asthma, allergic rhinitis, lung cancer, or any other significant respiratory disease, or were unable to understand the study-related questions.

The number of patients to be included in each country was pre-specified before enrolment. The contribution of each country was based on population size and proportionality to get representativeness based on population size.

The study was performed according to the principles of the Declaration of Helsinki. Local ethics committee approval (see Ethics Committees and Institutional Review Boards consulted at the end of the manuscript) was obtained before the start of the study. All patients were informed about the study and gave their written informed consent to participate. Data underlying the findings described in this manuscript may be obtained in accordance with AstraZeneca's data sharing policy described at https://astrazenecagrouptrials.pharmacm.com/ $\underline{\mathrm{ST} / \mathrm{Submission} / \mathrm{Disclosure} .}$ 


\section{Data Collection}

Data were recorded by the investigator using a standard case report form (CRF). The following sociodemographic and clinical data were collected: age, body mass index (BMI), smoking status, biomass exposure, spirometry, number of exacerbations during the previous 12 months, comorbidities, current maintenance treatment for COPD, vaccination (influenza, pneumococcal) in the last 12 months, current nonpharmacologic treatments for COPD (smoking cessation, pulmonary rehabilitation program, physical activity, oxygen therapy), and concomitant treatments.

The frequency of symptoms (breathlessness, phlegm, cough, wheezing, and chest tightness) in the last 7 days was recorded using a 5 -point scale $(0=$ not at all, $1=$ a little, $2=$ moderately, $3=$ very, and $4=$ extremely).

To assess symptom variability, the investigator recorded the following information "Did the patient experience daily variation in the severity of the symptoms" (Yes/No) for each symptom (breathlessness, phlegm, cough, wheezing, and chest tightness) if present in the last 7 days. The same information was recorded for weekly symptom variability.

Patients were also asked if they modified their COPD medication when experiencing symptom variability.

\section{Patient-Reported Outcomes}

The impact of symptom variability was assessed using patient questionnaires administered during the clinic visit, and ideally before any discussion with the physician in order to avoid influencing the patient's response. The questionnaires were self-explanatory and took about 15 to $20 \mathrm{~min}$ to complete. Certified translation of the English version of questionnaires and CRF was obtained in local languages for the study.

The Global Chest Symptoms Questionnaire (GCSQ) was used for evaluation of global chest symptoms during the day (in the morning, in the afternoon, in the evening, and at night). Patients had to answer two questions on how short of breath they felt and how tight did their chest feel most of the days in the last 7 days using a 5-point Likert scale $(0=$ not at all, $1=$ a little, $2=$ moderately, $3=$ very, and $4=$ extremely). The total score was calculated as the average score of the two questions and ranged between 0 and 4 , higher scores indicating more severe chest symptoms.

The Morning Activities and Symptoms Questionnaire (MASQ) was used for the evaluation of difficulty getting out of bed on most days of the past week. ${ }^{7}$ A modified 7-day recall version of the Capacity of Daily Living during the Morning (CDLM) questionnaire assessed the impact of COPD symptoms on activities during the first hours of the morning. ${ }^{8}$ The CDLM questionnaire consists of six questions that asked the patient if he/she was able to perform six common daily morning activities (i.e. washing themselves; drying themselves using a towel; getting dressed; eating breakfast; walking around the home early in the morning after taking medicine; and walking around your home later in the morning) and evaluated the degree of difficulty they normally experienced in most days of the past 7 days. The responses for each morning activity were used to calculate a score ranging from 0 (so difficult that the activity could not be carried out by the patient on their own) to 5 (activity was not at all difficult to carry out).

\section{Statistics}

Data were analyzed using SAS software version 9.2 (SAS institute, Cary, NC, USA). Statistics were mainly descriptive including mean, standard deviation (SD), median, minimal and maximal values for quantitative variables, and number and percentage for qualitative variables. Missing values were not computed. Study outcomes were calculated for each GOLD 2015 category and overall. To assess the impact of daily or weekly symptom variability on daily activities, the scores of the CDLM and MASQ questionnaires were compared between patients who did or did not report symptom variability. Between-group differences were tested using a Chi squared test, a Cochran Armitage test, or a Wilcoxon rank test where appropriate. The statistical significance level was two-sided and set at 0.05 .

A logistic regression analysis was performed to determine the factors associated with daily and weekly symptom variability. A logistic regression model adjusting for age, sex, and country was first performed for each factor tested including body mass index, current cigarette smoking, biomass exposure, time since COPD diagnosis, COPD GOLD category (C or D), number of COPD exacerbations during the last 12 months, comorbidities, depression/anxiety, chronic renal insufficiency, cancer, any other condition that significantly affects mobility (previous stroke or amputation), vaccination during the last year, and current COPD non-pharmacologic treatment (smoking cessation, pulmonary rehabilitation program, physical activity, oxygen therapy). Then, all factors (in addition to age, sex, and country) significantly associated with daily (respectively weekly) symptoms variability at the $20 \%$ level were included in the final model. Backward selection was used to select only the significant factors (at the 5\% level). 


\section{Results}

\section{Population Characteristics}

A total of 3253 patients were recruited and analyzed. Proportional to the country total population, 1143 (35.1\%) patients were recruited from Egypt, 810 (24.9\%) from Turkey, 711 (21.9\%) from Algeria, 314 (9.7\%) from South Africa, and 275 (8.4\%) from other countries. Patient sociodemographic and clinical characteristics are shown in Table 1. The mean age $( \pm \mathrm{SD})$ was $64.1 \pm 9.4$ years; $90.3 \%$ of patients were male; $35.9 \%$ were current smokers with a lower rate among GOLD C patients (29.0\%), and $25.2 \%$ reported exposure to biomass smoke. Overall, GOLD D patients had relatively more comorbidities than GOLD C patients $(62.1 \%$ versus $51.4 \%)$. The rate of patients with each comorbidity was higher in GOLD D patients than in GOLD C, especially hypertension $(41.8 \%$ versus $33.8 \%)$ and depression/anxiety $(7.1 \%$ versus $1.8 \%)$.

As summarized in Table 2, the mean $\mathrm{FEV}_{1}$ was 36.9 $\pm 9.7 \%$. The frequency of exacerbations during the last 12 months was $1.7 \pm 1.5$ (median 1.0), and was higher in GOLD D patients $(1.8 \pm 1.5$, median 2.0$) ; 79.6 \%$ in GOLD D had at least one exacerbation in the previous 12 months (38\% requiring hospitalization) compared to $54.1 \%$ in GOLD C with only $18.7 \%$ hospitalized.

Table I Patient Characteristics, by GOLD 2015 Category and for All Patients Combined

\begin{tabular}{|c|c|c|c|}
\hline & $\begin{array}{l}\text { GOLD C, } \\
\mathbf{N}=33 \text { I }\end{array}$ & $\begin{array}{l}\text { GOLD D, } \\
\mathrm{N}=2906\end{array}$ & $\begin{array}{l}\text { Total*, } \\
\text { N=3253 }\end{array}$ \\
\hline Age (years), mean $\pm S D$ & $63.7 \pm 9.5$ & $64.1 \pm 9.4$ & $64.1 \pm 9.4$ \\
\hline Males, n (\%) & $94.2 \%$ & $89.8 \%$ & $90.3 \%$ \\
\hline BMI $\left(\mathrm{kg} / \mathrm{m}^{2}\right)$, mean $\pm \mathrm{SD}$ & $26.1 \pm 5.2$ & $26.3 \pm 5.9$ & $26.3 \pm 5.8$ \\
\hline Active smokers, n (\%) & $29.0 \%$ & $36.6 \%$ & $35.9 \%$ \\
\hline Ex-smokers, n (\%) & $65.3 \%$ & $59.0 \%$ & $35.9 \%$ \\
\hline Biomass exposure, $n$ (\%) & $29.2 \%$ & $24.7 \%$ & $25.2 \%$ \\
\hline \multicolumn{4}{|l|}{ Main comorbidities } \\
\hline Hypertension, n (\%) & $33.8 \%$ & $41.8 \%$ & $41.0 \%$ \\
\hline Diabetes mellitus, n (\%) & $18.3 \%$ & $21.8 \%$ & $21.4 \%$ \\
\hline Hyperlipidemia, n (\%) & $13.7 \%$ & $16.2 \%$ & $15.9 \%$ \\
\hline Ischemic heart disease, $\mathrm{n}(\%)$ & $13.7 \%$ & $14.8 \%$ & $14.7 \%$ \\
\hline Heart failure, n (\%) & $4.6 \%$ & $7.1 \%$ & $6.8 \%$ \\
\hline Depression/anxiety, n (\%) & $1.8 \%$ & $7.1 \%$ & $6.6 \%$ \\
\hline Arrhythmia, n (\%) & $4.6 \%$ & $5.7 \%$ & $5.6 \%$ \\
\hline Osteoporosis, n (\%) & $1.5 \%$ & $5.8 \%$ & $5.3 \%$ \\
\hline
\end{tabular}

Note: *GOLD 2015 category was not known in 16 patients.
Table 2 Disease-Related Variables, by GOLD 2015 Category and for All Patients Combined

\begin{tabular}{|c|c|c|c|}
\hline & $\begin{array}{l}\text { GOLD C, } \\
N=331\end{array}$ & $\begin{array}{l}\text { GOLD D, } \\
N=2906\end{array}$ & $\begin{array}{l}\text { Total, } \\
N=3237\end{array}$ \\
\hline $\begin{array}{l}\text { Time from diagnosis } \\
\text { (years) }\end{array}$ & $6.6 \pm 5.6$ & $9.3 \pm 7.1$ & $9.0 \pm 7.0$ \\
\hline FEV (\% predicted) & $39.5 \pm 9.9$ & $36.5 \pm 9.6$ & $36.9 \pm 9.7$ \\
\hline FVC (\% predicted) & $62.7 \pm 16.0$ & $59.1 \pm 16.1$ & $59.4 \pm 16.1$ \\
\hline $\mathrm{FEV}_{1} / \mathrm{FVC}(\%)$ & $53.9 \pm 12.1$ & $53.6 \pm 12.8$ & $53.6 \pm 12.7$ \\
\hline Number of exacerbations* & $0.9 \pm 1.1$ & $1.8 \pm 1.5$ & $1.7 \pm 1.5$ \\
\hline At least one exacerbation* & $179(54.1 \%)$ & $2313(79.6 \%)$ & $2492(77.0 \%)$ \\
\hline $\begin{array}{l}\text { Treatment with } \\
\text { antibiotics }\end{array}$ & 164 (49.5\%) & $2125(73.1 \%)$ & $2289(70.7 \%)$ \\
\hline $\begin{array}{l}\text { Treatment with oral } \\
\text { steroids }\end{array}$ & 118 (35.6\%) & $1699(58.5 \%)$ & $1817(56.1 \%)$ \\
\hline Emergency room visit & 94 (28.4\%) & $1632(56.2 \%)$ & $1726(53.3 \%)$ \\
\hline Hospitalization & $62(18.7 \%)$ & $1104(38.0 \%)$ & $1166(36.0 \%)$ \\
\hline $\begin{array}{l}\text { Non-invasive mechanical } \\
\text { ventilation }\end{array}$ & $8(2.4 \%)$ & $275(9.5 \%)$ & $283(8.7 \%)$ \\
\hline Mechanical ventilation & $8(2.4 \%)$ & $155(5.3 \%)$ & $163(5.0 \%)$ \\
\hline $\begin{array}{l}\text { Invasive mechanical } \\
\text { ventilation }\end{array}$ & - & $58(2.0 \%)$ & $58(1.8 \%)$ \\
\hline CAT score & $6.8 \pm 2.1$ & $22.0 \pm 6.8$ & $20.5 \pm 7.9$ \\
\hline
\end{tabular}

Notes: *In the last 12 months (for patients to be included in the study, the patients were to be stable i.e. no exacerbation within the last 3 months). Data are mean $\pm S D$ or number $(\%)$ of patients.

Current maintenance treatments are shown in Table 3. The most frequent treatments were LABA plus ICS (as

Table 3 COPD Maintenance Treatment, by GOLD 2015 Category

\begin{tabular}{|l|l|l|}
\hline & $\begin{array}{l}\text { GOLD C, } \\
\text { N=33 I }\end{array}$ & $\begin{array}{l}\text { GOLD D, } \\
\text { N=2906 }\end{array}$ \\
\hline Pharmacological Treatments & & \\
SABA or SAMA & $60.4 \%$ & $58.3 \%$ \\
LABA & $12.1 \%$ & $18.6 \%$ \\
LAMA & $65.0 \%$ & $56.7 \%$ \\
LABA + LAMA & $6.0 \%$ & $10.2 \%$ \\
ICS & $8.2 \%$ & $16.5 \%$ \\
ICS + LABA (Fixed-dose combination) & $61.0 \%$ & $52.3 \%$ \\
ICS + LABA (Free combination) & $14.2 \%$ & $15.2 \%$ \\
Methylxanthines & $13.9 \%$ & $27.8 \%$ \\
Antibiotics & $3.3 \%$ & $6.1 \%$ \\
\hline Non-Pharmacological Treatments & & \\
Smoking cessation & $28.6 \%$ & $29.3 \%$ \\
Pulmonary rehabilitation program & $5.2 \%$ & $6.6 \%$ \\
Physical activity & $20.5 \%$ & $16.7 \%$ \\
Oxygen therapy & $7.3 \%$ & $15.5 \%$ \\
\hline
\end{tabular}

Notes: Data are percentage of patients. Treatments as recorded in the CRF. Several treatments (association) could be ticked.

Abbreviations: ICS, inhaled corticosteroid; LABA, long-acting beta agonist; LAMA, long-acting muscarinic antagonist; SABA, short-acting beta agonist; SAMA, short-acting muscarinic antagonist. 
fixed or free combination) prescribed in $68.3 \%$ of patients, LAMA or LAMA plus LABA in $67.4 \%$ of patients, and SABA or SAMA in $58.5 \%$ of patients. In addition, oxygen therapy was prescribed in $15.5 \%$ of GOLD D patients versus $7.3 \%$ of GOLD C patients. Fewer GOLD D (39.4\%) patients had received influenza vaccination during the last year compared to GOLD C patients $(48.0 \%)$, although a greater proportion had received pneumococcal polysaccharide vaccine (11.3\% versus $8.9 \%)$.

\section{Severity of Symptoms}

As shown in Figure 1, breathlessness in the last 7 days was reported in $84.6 \%$ of patients, phlegm in $73.6 \%$, cough in $75.4 \%$, wheezing in $66.9 \%$, and chest tightness in $72.1 \%$. The severity was mild in $26 \%$ to $34 \%$ of patients, moderate in $27 \%$ to $36 \%$, and very or extremely severe in $12 \%$ to $22 \%$ of patients.

\section{Daily and Weekly Symptoms Variability}

When patients were asked about daily and weekly variability, $83.4 \%$ reported at least one symptom with daily variability including breathlessness (64.6\%), phlegm $(52.9 \%)$, cough $(54.8 \%)$, wheezing $(52.9 \%)$, or chest tightness $(56.7 \%)$. This was significantly higher in GOLD $\mathrm{D}$ than GOLD C patients (83.9\% versus $76.9 \%, \mathrm{p}=0.012)$.

The proportion of patients with daily symptom variability for each symptom is shown in Figure 2A. This variability was significantly $(\mathrm{p}<0.001)$ higher in GOLD D patients for breathlessness $(65.7 \%$ versus $51.0 \%)$, cough $(55.9 \%$ versus $40.7 \%$ ), wheezing $(54.3 \%$ versus $33.6 \%)$, and chest tightness (58.7\% versus $30.8 \%$ ), and higher but not significantly for phlegm (53.5\% versus $46.7 \%$, $\mathrm{p}=0.092)$. Similar results were shown for weekly symptom variability (Figure 2B).

Factors significantly associated with variation of each symptom in multivariate logistic regression analysis are

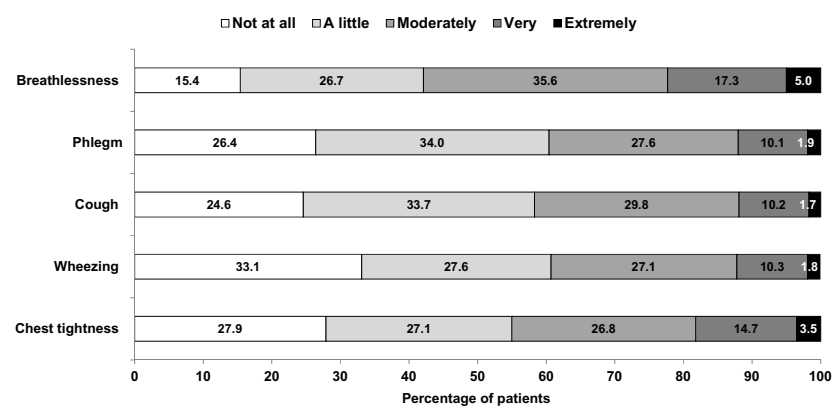

Figure I COPD symptoms in the last 7 days (all patients). At the inclusion visit, each patient was asked to report the presence and the severity of each symptom in the last 7 days.

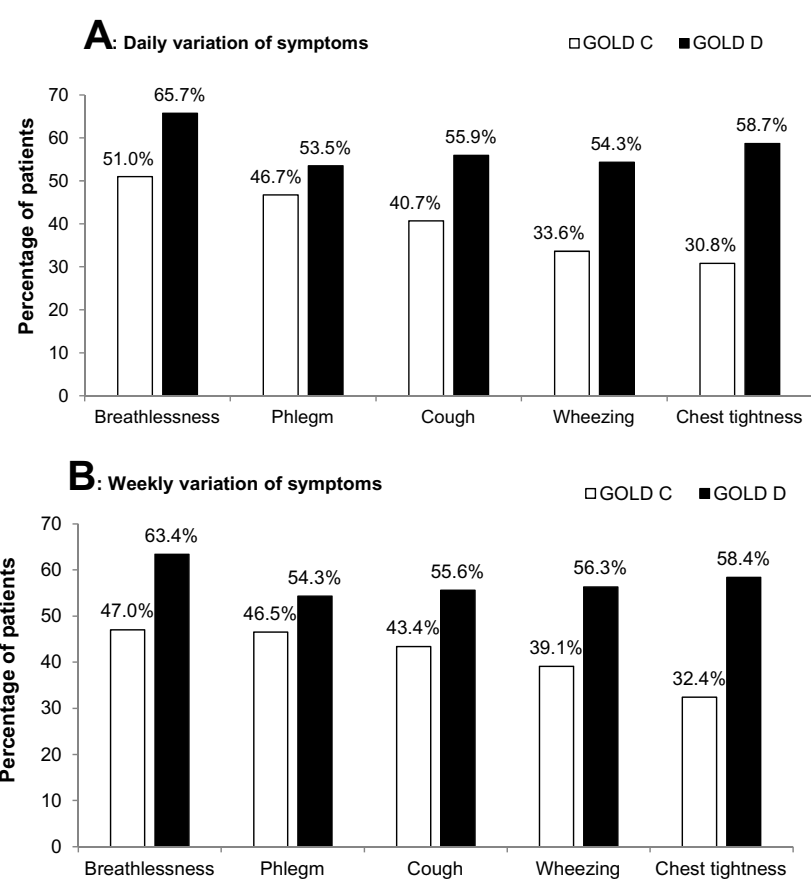

Figure 2 Daily and weekly variation of symptoms. Patients with COPD symptoms during the last 7 days were asked if they experienced or not daily/weekly variation in the severity of symptoms. Panel A: Daily variation. Panel B: Weekly variation.

shown in Table 4. Overall, the number of exacerbations in the previous year and COPD GOLD D were consistently associated with a higher risk of daily symptom variability, independently of other variables. Smoking cessation was associated with lower risk of daily symptom variability. Similar results were obtained in the analysis of weekly symptom variability (data not shown), except that cigarette smoking was a significant factor for breathlessness variability $(\mathrm{OR}=1.33,95 \% \mathrm{CI}: 1.08,1.63, \mathrm{p}<0.0071)$, phlegm production variability $(\mathrm{OR}=1.38,95 \% \mathrm{CI}: 1.13,1.70, \mathrm{p}<0.0018)$, and chest tightness variability $(\mathrm{OR}=1.27,95 \% \mathrm{CI}: 1.03,1.57$, $\mathrm{p}=0.0227$ ).

\section{Global Chest Symptoms Questionnaire (GCSQ)}

Overall, regardless of the GOLD category, the mean GCSQ score ranged between 0 and 4 without relevant difference according to time of day (morning, afternoon, evening, and night), especially in GOLD $C$ patients $(0.4 \pm 0.5$ or \pm 0.6 at each time point). The GCSQ score was significantly higher $(\mathrm{p}<0.001)$ in GOLD D patients than in GOLD C patients at each timepoint (morning, afternoon, evening, and night) (Table 5). In GOLD D, the GCSQ score was significantly higher at night $(1.6 \pm 1.2, \mathrm{p}<0.001)$ and in the morning $(1.5$ $\pm 1.0, \mathrm{p}<0.001)$ and lower in the afternoon $(1.3 \pm 0.9)$. 
Table 4 Factors Associated with Daily Variation of Each COPD Symptom

\begin{tabular}{|c|c|c|c|c|}
\hline $\begin{array}{l}\text { Daily Variation of } \\
\text { Symptoms }\end{array}$ & $\mathbf{N}$ & Effect & Adjusted OR $[95 \% \mathrm{CI}]$ & $\operatorname{Pr}>$ Khi-2 \\
\hline Breathlessness & 1998 & $\begin{array}{l}\text { Number of exacerbations* } \\
\text { GOLD category (D versus C) } \\
\text { Smoking cessation (No versus Yes) } \\
\text { Hypertension (Yes versus No) } \\
\text { COPD diagnosis (years)* } \\
\text { Oxygen therapy (No versus Yes) }\end{array}$ & $\begin{array}{l}1.26[1.15 ; 1.37] \\
2.15[1.51 ; 3.07] \\
0.67[0.54 ; 0.85] \\
1.34[1.09 ; 1.64] \\
1.02[1 ; 1.03] \\
1.43[1.05 ; 1.93]\end{array}$ & $\begin{array}{l}<0.0001 \\
<0.0001 \\
0.0008 \\
0.0054 \\
0.0171 \\
0.0219\end{array}$ \\
\hline Phlegm & 1812 & $\begin{array}{l}\text { Number of exacerbations* } \\
\text { COPD diagnosis (years)* } \\
\text { Hyperlipidaemia (Yes versus No) }\end{array}$ & $\begin{array}{l}1.28[1.18 ; 1.38] \\
1.02[1.01 ; 1.04] \\
1.3[1 ; 1.69]\end{array}$ & $\begin{array}{l}<0.0001 \\
0.0010 \\
0.0488\end{array}$ \\
\hline Cough & 1856 & $\begin{array}{l}\text { Number of exacerbations* } \\
\text { Smoking cessation (Yes versus No) } \\
\text { GOLD category (D versus C) } \\
\text { COPD diagnosis (years)* } \\
\text { Other conditions that significantly affect mobility (Yes } \\
\text { versus No) }\end{array}$ & $\begin{array}{l}1.26[1.17 ; 1.36] \\
0.63[0.5 ; 0.79] \\
\text { I.86 [1.28; } 2.69] \\
1.02[1.01 ; 1.04] \\
0.4[0.17 ; 0.91]\end{array}$ & $\begin{array}{l}<0.0001 \\
<0.0001 \\
0.0011 \\
0.0032 \\
0.0285\end{array}$ \\
\hline Wheezing & 1715 & $\begin{array}{l}\text { COPD GOLD category (D versus C) } \\
\text { COPD diagnosis (years)* } \\
\text { Number of exacerbations* } \\
\text { Biomass exposure (Yes versus No) } \\
\text { Pulm. Rehabilit. Progr. (Yes versus No) } \\
\text { Cigarette smoker (Yes versus No) } \\
\text { Ischemic heart disease (Yes versus No) } \\
\text { Smoking cessation (Yes versus No) }\end{array}$ & $\begin{array}{l}2.54[1.65 ; 3.92] \\
1.03[1.02 ; 1.05] \\
1.17[1.08 ; 1.27] \\
1.47[1.15 ; 1.89] \\
1.87[1.22 ; 2.85] \\
1.35[1.08 ; 1.69] \\
1.43[1.07 ; 1.91] \\
0.76[0.59 ; 0.99]\end{array}$ & $\begin{array}{l}<0.0001 \\
<0.0001 \\
0.0001 \\
0.0024 \\
0.0040 \\
0.0087 \\
0.0167 \\
0.0406\end{array}$ \\
\hline Chest tightness & 1763 & $\begin{array}{l}\text { GOLD category (D versus C) } \\
\text { Smoking cessation (Yes versus No) } \\
\text { Number of exacerbations* } \\
\text { COPD diagnosis (years)* } \\
\text { Hypertension (Yes versus No) } \\
\text { Ischemic heart disease (Yes versus No) } \\
\text { Biomass exposure (Yes versus No) } \\
\text { Oxygen therapy (Yes versus No) }\end{array}$ & $\begin{array}{l}3.11[2.05 ; 4.74] \\
0.66[0.52 ; 0.84] \\
1.14[1.06 ; 1.24] \\
1.02[1 ; 1.04] \\
1.3[1.05 ; 1.61] \\
1.39[1.04 ; 1.86] \\
1.31[1.02 ; 1.69] \\
1.38[1.02 ; 1.86]\end{array}$ & $\begin{array}{l}<0.0001 \\
0.0006 \\
0.0011 \\
0.0094 \\
0.0166 \\
0.0283 \\
0.0323 \\
0.0342\end{array}$ \\
\hline
\end{tabular}

Note: *Continuous variable.

Abbreviation: $\mathrm{N}$, number of observations.

\section{Difficulty to Get Out of Bed (MASQ)}

As assessed by the MASQ questionnaire, the proportion of patients with difficulty getting out of bed in the morning was significantly higher in GOLD D patients than in GOLD C patients $(60.0 \%$ versus $13.6 \%, \mathrm{p}<0.0001)$. The level of difficulty getting out of bed was also significantly more severe in GOLD D patients (Figure 3).

The difficulty to get out of bed was assessed using the MASQ questionnaire in patients with and without symptom variability. As shown in Figure 4, patients with daily variation of chest tightness had more severe difficulty getting out of bed in the morning $(\mathrm{p}<0.0001)$. Similar results were shown for daily variation in wheezing $(p<0.0001)$ and cough $(p=0.0081)$, but not breathlessness $(p=0.1059)$ or phlegm $(p=0.9396)$. Among patients who reported daily variation of chest tightness, $38.2 \%$ had at least moderate difficulty getting out of bed in the morning (versus 29.1\%) (Figure 4). Similar percentages were obtained for cough $(34.7 \%$ versus $29.2 \%)$ and wheezing (38.5\% versus $28.6 \%$ ).

There were statistically significant associations between difficulty getting out of bed and weekly variation in breathlessness $(p=0.0079)$, wheezing $(p=0.0064)$, and chest tightness $(\mathrm{p}<0.0001)$. 
Table 5 GCSQ at Each Time Point in GOLD C and GOLD D

\begin{tabular}{|c|c|c|c|c|c|}
\hline & & Morning & Afternoon & Evening & Night \\
\hline GOLD C & $\begin{array}{l}\text { N } \\
\text { Mean } \pm S D \\
\text { Median } \\
\text { Min.; Max }\end{array}$ & $\begin{array}{l}325 \\
0.4 \pm 0.6 \\
0 \\
0 ; 4\end{array}$ & $\begin{array}{l}322 \\
0.4 \pm 0.5 \\
0 \\
0 ; 4\end{array}$ & $\begin{array}{l}322 \\
0.4 \pm 0.6 \\
0 \\
0 ; 4\end{array}$ & $\begin{array}{l}325 \\
0.4 \pm 0.6 \\
0 \\
0 ; 4\end{array}$ \\
\hline GOLD D & $\begin{array}{l}\mathrm{N} \\
\text { Mean } \pm S D \\
\text { Median } \\
\text { Min.; Max }\end{array}$ & $\begin{array}{l}2840 \\
1.5 \pm 1.0 \\
1.5 \\
0 ; 4\end{array}$ & $\begin{array}{l}2802 \\
1.3 \pm 0.9 \\
1.0 \\
0 ; 4\end{array}$ & $\begin{array}{l}2803 \\
1.5 \pm 1.0 \\
1.4 \\
0 ; 4\end{array}$ & $\begin{array}{l}2801 \\
1.6 \pm 1.2 \\
1.5 \\
0 ; 4\end{array}$ \\
\hline P-value* & & $<0.001$ & $<0.001$ & $<0.001$ & $<0.001$ \\
\hline
\end{tabular}

Note: *Wilcoxon rank test.

\section{Capacity of Daily Living During the Morning Activities (CDLM)}

The CDLM questionnaire was used to determine the impact of chest symptoms on usual morning activities during the past 7 days (Table 6). The mean CDLM global score ranged between 0 (activity so difficult that the patient was not able to carry out on their own) and 5 (no difficulty) and was worse $(\mathrm{p}<0.0001)$ in GOLD D $(3.5 \pm 1.1)$ than in GOLD C (4.6 \pm 3.5$)$ patients. Walking was the most severely affected activity in the morning; $51.3 \%$ of patients in

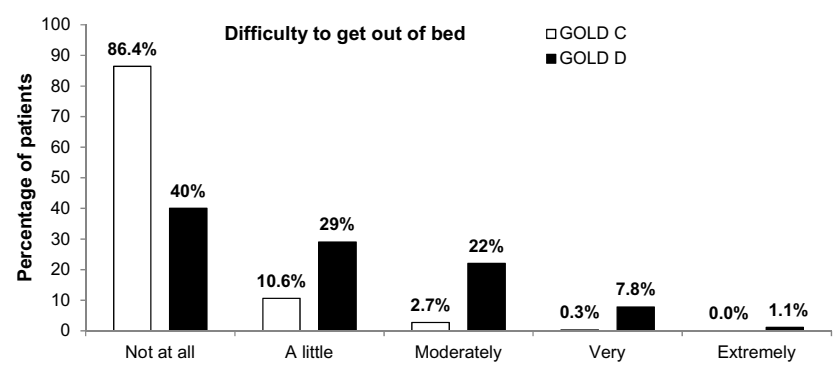

Figure 3 Therapeutic behavior in response to symptom variability. The patients were asked if they modify their COPD medication when experiencing symptom variability over the past 7 days (more than one response possible). Differences between groups were assessed using a Chi square test.

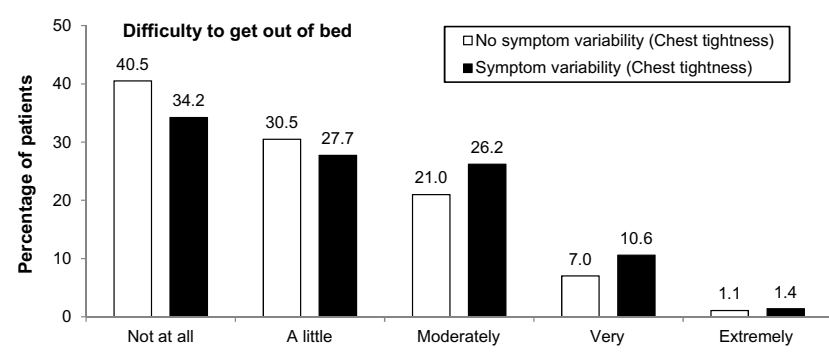

Figure 4 Difficulty to get out of bed in the morning (MASQ). Patients were asked: "Thinking of your chest condition in the past 7 days, how difficult was it for you to get out of bed in the morning?"
Table 6 CDLM Global Score and Subscores by GOLD 2015 Category

\begin{tabular}{|l|l|l|l|}
\hline & $\begin{array}{l}\text { GOLD C, } \\
\text { N=33 I }\end{array}$ & $\begin{array}{l}\text { GOLD D, } \\
\text { N=2906 }\end{array}$ & P-value* \\
\hline CDLM global score & $4.6 \pm 3.5(5)$ & $3.5 \pm 1 . I(4)$ & $<0.000$ I \\
Wash yourself & $4.7 \pm 0.9(5)$ & $3.4 \pm 1.6(4)$ & $<0.000$ I \\
$\begin{array}{l}\text { Dry yourself with a towel } \\
\text { Get dressed in the } \\
\text { morning }\end{array}$ & $4.9 \pm 0.4(5)$ & $3.9 \pm 1.2(4)$ & $<0.000$ I \\
$\begin{array}{l}\text { Eat breakfast in the } \\
\text { morning }\end{array}$ & $4.8 \pm 0.5(5)$ & $3.8 \pm 1.3(4)$ & $<0.000 \mathrm{I}$ \\
$\begin{array}{l}\text { Walk around home early } \\
\text { in the morning, after } \\
\text { taking medicine }\end{array}$ & $4.2 \pm 1.6(5)$ & $2.8 \pm 2.0(3)$ & $<0.000 \mathrm{I}$ \\
$\begin{array}{l}\text { Walk around home later } \\
\text { in the morning }\end{array}$ & $4.1 \pm 1.6(5)$ & $2.8 \pm 1.9(3)$ & $<0.000 \mathrm{I}$ \\
\hline
\end{tabular}

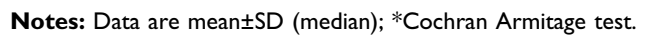

GOLD D versus $15.2 \%$ in GOLD C reported moderate to severe difficulties for walking around home early (after taking medicine) in the morning, and $51.8 \%$ versus $15.5 \%$, respectively, later in the morning.

Overall, the CDLM global score was not significantly different between patients with and without daily symptom variability $(3.6 \pm 1.2$ and $3.6 \pm 1.0, \mathrm{p}=0.548)$. However, according to the symptom, some morning activities were shown to be significantly affected by daily variability (Table 7). Wheezing and chest tightness, for example, were the symptoms which daily variability had the most consistent impact on morning activities. By comparison, there was no statistically significant effect of weekly symptom variability on CDLM score or subscores (data not shown).

\section{Therapeutic Behavior}

The therapeutic behavior of patients regarding symptom variability over the past 7 days is described in Figure 5. Overall, 
Table 7 Morning Activities Significantly Affected by Daily Symptom Variability

\begin{tabular}{|c|c|c|c|}
\hline \multirow[t]{2}{*}{ Mean ( $\pm S D)$ CDLM Subscore } & \multicolumn{2}{|c|}{ Daily Symptom Variability } & \multirow[t]{2}{*}{ P-value* } \\
\hline & No & Yes & \\
\hline Daily Variation in Breathlessness & $(\mathrm{N}=959)$ & $(N=1749)$ & \\
\hline Wash yourself & $3.5 \pm 1.5$ & $3.3 \pm 1.6$ & 0.003 \\
\hline Walk around home later in the morning & $3.1 \pm 1.9$ & $2.8 \pm 2.0$ & 0.002 \\
\hline Daily variation in Cough & $(\mathrm{N}=1125)$ & $(N=1366)$ & \\
\hline Wash yourself & $3.5 \pm 1.5$ & $3.3 \pm 1.6$ & 0.004 \\
\hline Get dressed in the morning & $3.9 \pm 1.2$ & $3.7 \pm 1.4$ & 0.014 \\
\hline Eat breakfast in the morning & $4.0 \pm 1.5$ & $3.8 \pm 1.7$ & $<0.001$ \\
\hline Walk around home early in the morning, after taking medicine & $2.9 \pm 2.0$ & $2.7 \pm 2.1$ & 0.025 \\
\hline Daily variation in wheezing & $(N=1075)$ & $(N=1204)$ & \\
\hline Wash yourself & $3.5 \pm 1.5$ & $3.1 \pm 1.6$ & $<0.001$ \\
\hline Dry yourself with a towel & $3.9 \pm 1.2$ & $3.8 \pm 1.2$ & $<0.001$ \\
\hline Get dressed in the morning & $3.8 \pm 1.2$ & $3.6 \pm 1.4$ & $<0.001$ \\
\hline Eat breakfast in the morning & $4.0 \pm 1.5$ & $3.7 \pm 1.7$ & $<0.001$ \\
\hline Walk around home early in the morning, after taking medicine & $2.9 \pm 1.9$ & $2.5 \pm 2.0$ & $<0.001$ \\
\hline Walk around home later in the morning & $3.0 \pm 1.8$ & $2.6 \pm 2.0$ & $<0.001$ \\
\hline Daily variation in chest tightness & $(N=1037)$ & $(N=1360)$ & \\
\hline Wash yourself & $3.6 \pm 1.5$ & $3.2 \pm 1.6$ & $<0.001$ \\
\hline Dry yourself with a towel & $4.0 \pm 1.2$ & $3.7 \pm 1.2$ & $<0.001$ \\
\hline Get dressed in the morning & $3.8 \pm 1.2$ & $3.6 \pm 1.3$ & $<0.001$ \\
\hline Eat breakfast in the morning & $4.0 \pm 1.6$ & $3.8 \pm 1.7$ & $<0.001$ \\
\hline Walk around home early in the morning, after taking medicine & $2.9 \pm 2.0$ & $2.6 \pm 2.0$ & $<0.001$ \\
\hline Walk around home later in the morning & $3.0 \pm 1.9$ & $2.6 \pm 2.0$ & $<0.001$ \\
\hline
\end{tabular}

Notes: Data are means $\pm S D$; *Wilcoxon rank test.

a high proportion of patients $(55.2 \%)$ reported that they continued to use their medications exactly in the same way despite symptom variability. This was significantly higher in GOLD $C$ than in GOLD D patients $(62.7 \%$ versus $54.5 \%$, $\mathrm{p}=0.005)$. On the other hand, $15.9 \%$ versus $27.9 \%(\mathrm{p}<0.001)$ of patients with GOLD C and GOLD disease, respectively, declared to change the dose and/or frequency of their maintenance medication. The use of short-acting bronchodilators,

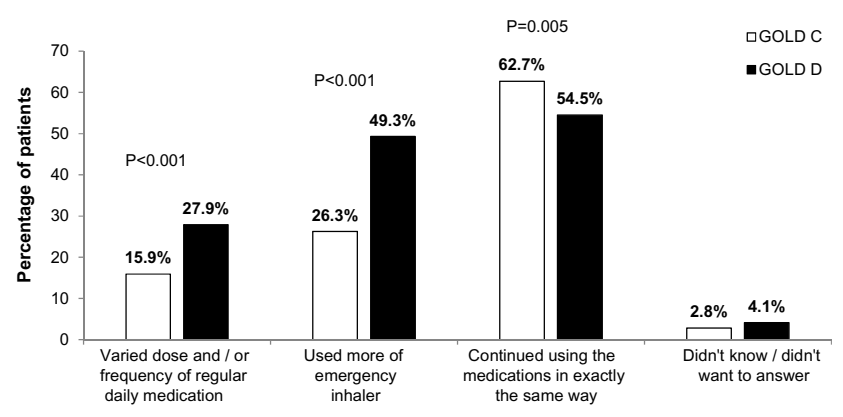

Figure 5 Daily symptom variability and difficulty to get out of bed (MASQ). The difficulty to get out of bed was assessed using the MASQ questionnaire in patients with and without symptom variability. Patients with daily variation of chest tightness had more severe difficulty to get out of bed in the morning $(p<0.000 \mathrm{I})$. as rescue medication, was also more frequent among patients with GOLD D disease (49.3\% versus $26.3 \%$, $<<0.001)$.

\section{Discussion}

To the best of our knowledge, this is the first study investigating the burden of symptoms and their variability in a large cohort of stable severe COPD patients in the MEA region. The COPVAR study indicates that stable COPD patients in both GOLD (2015) C and D stages have significant chest symptom variability that considerably impacts morning activities, especially in GOLD D patients as assessed using the CDLM and MASQ questionnaire.

The study involved 3253 patients. This included a small percentage of GOLD C patients (10\%), which is consistent with the proportion in previous reports. ${ }^{9,10}$ The demographic characteristics in GOLD C/D patients in this study were similar to global trends in which most affected people were men with a mean age of 64 years, and a smoking rate of $36 \%$. High smoking rate in severely impaired population is not surprising as compared with other studies. $^{11-13}$ Although smoking cessation is offered 
to almost every COPD patient, a high proportion of them continue to smoke and $40 \%$ do not try to quit, which is consistent with other studies. ${ }^{14}$ Biomass smoke exposure is a common feature among COPD patients, particularly in developing countries. ${ }^{15}$ In the COPVAR study, biomass smoke exposure rate was approximately $25 \%$, which is lower than a rate $(47 \%)$ previously reported in Turkey. ${ }^{16}$ Difference in biomass exposure according to geographic localization is likely. Cardiovascular comorbidities were also commonly reported in COPVAR, consistent with other studies. ${ }^{17,18}$ Another observational study in Turkey showed that $13.2 \%$ of GOLD $\mathrm{C}$ and $51.4 \%$ of GOLD $\mathrm{D}$ patients had at least one hospitalization history for COPD, which is quite similar in COPVAR $(18.7 \%$ and $38.0 \%$, respectively). ${ }^{19}$

In COPVAR, a relevant proportion of patients experienced moderate to severe symptoms in the last 7 days despite maintenance treatment. Breathlessness was the most common symptom ( $84.6 \%$ of patients) followed by coughing (75.4\%), phlegm production (73.6\%), chest tightness $(72.1 \%)$, and wheezing $(66.9 \%)$. The prevalence of these symptoms was higher compared to other stable COPD patients, especially wheezing and chest tightness, reported in $41.4 \%$ and $32.9 \%$, respectively, in another study. $^{20}$ The high prevalence of wheezing may indicate that some patients with features of asthma were enrolled, although this was an exclusion criteria for the study.

The COPVAR study showed a high heterogeneity in COPD symptom severity in both groups of patients. Some patients had no symptoms at all while others had very severe or extremely severe symptoms. This may reflect different phenotypes within each GOLD category, as suggested previously. $^{21,22}$

This study showed that a high proportion of patients $(>80 \%)$ perceived daily and weekly variability in symptoms. Such variability was shown to be associated with various factors including comorbidities, disease factors including COPD diagnosis, smoking status, and nonpharmacological intervention. However, the most consistent cofactors associated with symptom variability were the number of exacerbations during the last year and patients in the GOLD D category. In accordance with previous studies, such associations suggest an increased risk of exacerbations and a worse disease prognosis in patients with symptom variability. ${ }^{23}$ In addition, GOLD D patients in COPVAR had experienced a significantly higher frequency of exacerbations in the previous 12 months including hospitalization than GOLD $\mathrm{C}$ patients, which may be related to higher symptom variability in GOLD D patients.

Otherwise, the study also showed a statistically significant association between symptom variability and cigarette smoking (higher risk) and smoking cessation (lower risk). Gender was not found as a significant cofactor in the logistic regression analysis. Thus, there is likely no difference between males and females in symptom variability in this population.

Findings of the COPVAR study show that GOLD $\mathrm{C}$ and $\mathrm{D}$ patients perceived different burden of symptoms, levels of variability, and related impact on daily activities. As confirmed recently, patients with stable COPD commonly experience daily symptom variability with the morning being the most troublesome time of the day followed by night. ${ }^{24}$ It was reported that the proportion of patients with breathlessness increased from nighttime through the morning and into the daytime, whereas coughing and bringing up phlegm or mucus were most common early in the morning. ${ }^{20}$ In COPVAR, the variability in breathlessness and chest tightness (assessed using the GCSQ questionnaire) was quite similar at each time of the day, especially in GOLD C patients. Nevertheless, it should be noted that in GOLD D patients the GCSQ was higher at night and morning than in the afternoon. Although differences between GCSQ scores at different times of the day may appear quite small, a minimal difference of 0.15 points in GSQG score may be considered as clinically relevant. ${ }^{25,26}$

As suggested, patients with COPD may experience diurnal variation in lung function, which may explain daytime symptom variability, including breathlessness and chest tightness. $^{27}$

The COPVAR study also confirmed that COPD symptoms, especially morning symptoms, are associated with a clinically meaningful decline in quality of life, which could impact overall health status and prognosis as reported previously. ${ }^{28}$ COPD symptoms progressively compromise the patient's ability to function normally in terms of their day-to-day activities.

Difficulty getting out of bed is particularly problematic in patients with severe COPD. ${ }^{27}$ Here, using the MASQ questionnaire, we found that the difficulty in getting out of bed was significantly associated with daily symptom variability in cough, wheezing, and chest tightness and with weekly variability in breathlessness, wheezing, and chest tightness. Overall, using the CDLM questionnaire, wheezing and chest tightness variability seems to have a more 
relevant impact on morning activities especially walking home early (or later) in the morning than other symptoms. Chest tightness was reported as more prominent in the morning compared with other parts of the day. ${ }^{27}$ Chest tightness (compared to breathlessness, phlegm, or cough) is also a strong predictive factor for the presence of morning symptoms limiting morning activities in patients with severe airflow limitation. ${ }^{27}$ In COPVAR, the greatest difference in daily symptom variability between GOLD $\mathrm{C}$ and GOLD D patients was shown for chest tightness (30.8\% versus $58.7 \%)$.

By definition, GOLD C patients have fewer symptoms than GOLD D. The COPVAR study showed that GOLD D patients are more affected than GOLD C in the morning activities. On average, GOLD C patients were rather mildly impacted on their morning activities as assessed by the CDLM questionnaire. On the contrary, GOLD D patients were more severely affected, especially walking around early or later in the morning. This is consistent with a recent study showing that the CAT score was independently associated with the CDLM score and thus with the impact on morning activities in COPD patients. ${ }^{29}$ Thus, although both GOLD C and GOLD D patients reported to perceive daily and weekly symptom variability, they were differently affected in the morning activities. GOLD D patients had a higher risk of symptom variability than GOLD C as shown by the regression analyses and are more affected in their morning activities.

In COPVAR, despite symptom variability, around 50\% of patients did not change their treatment which is consistent with previous reports. ${ }^{3}$ Despite the recognized importance of addressing COPD symptoms in the overall management of $\mathrm{COPD},{ }^{30}$ the results of COPVAR suggest that the maintenance treatments and/or adherence to treatment are insufficient in a number of patients and a treatment strategy to minimize the impact of this variation should be provided or reinforced. Improved control of patients' morning symptoms may lead to a substantial reduction in COPD impact and frequency of exacerbations, and enable patients to increase daily activities, particularly early morning activities, as previously suggested. ${ }^{31}$

We acknowledge that this study has several limitations. The results showed that a high proportion of patients had wheezing, which is consistent with the concomitant asthmatic disease. It is not known to what extent patients with features of asthma were included in this study given that asthma was an exclusion criterion. This study confirmed that stable severe COPD patients with frequent exacerbations perceived daily and weekly symptom variability, impacting morning activities. It is known that sputum and cough have the highest circadian variability in COPD patients, ${ }^{5}$ but this was not investigated. Lung function has also an impact on morning activities, including the CDLM score as reported recently, ${ }^{25}$ and was not systematically measured at the study visit. Only $\mathrm{FEV}_{1}$ and FVC were to be documented in the last 12 months. Symptom variability was also reported according to seasons, ${ }^{28}$ and this was not analyzed in this multi-country study.

It is also clear from this study that patients who did not report symptom variability are also highly impacted in the morning activities, and the association between CDLM and MASQ scores and symptom variability could be adjusted by confounding factors which remains to be identified, especially when the impact on morning activities is severe. In this study, most patients $(>80 \%)$ were males; thus, studies with greater numbers of female patients are required to be able to confidently evaluate symptoms in female patients with COPD from this region. Poor treatment adherence was recently reported in Turkey and Saudi Arabia, ${ }^{32}$ and may explain the symptom variability in this study. There were additional differences between patients' characteristics in each country including age, sex ratio, comorbidities, and symptoms (data not shown), which might account for inter-country differences. The logistic regression of symptom variability showed a statistically significant country effect $(p<0.0001)$ suggesting different associated factors according to countries (data not shown). Nevertheless, there were consistent data from each country showing that the number of exacerbations in the previous year, smoking cessation, and GOLD D (versus GOLD C) are independent predictors of symptom variability (data not shown).

\section{Conclusion}

As reported in other populations, the majority of patients with severe COPD in the MEA region perceive significant daily or weekly variability in their respiratory symptoms, interfering with morning activities. It is important to assess symptom variability in these patients to improve disease management and appropriate interventions to address variability in symptoms and exacerbation prevention. 


\section{Ethics Committee (EC) or Institutional Review Board (IRB) Consulted}

Egypt (Central Directorate of Research \& Health Development, Ministry of Health \& Population, Cairo); UAE (Zayed Military Hospital Ethics Committee; Rashid Hospital, DHA, Dubai Scientific Research EC; Al Ain Hospital Research \& Ethics Governance Committee); Kuwait (MOH-Kuwait); Qatar (Hamad Medical Center Institutional Review Board); Turkey (Gazi University Clinical Trials Ethics Committee); Kingdom of Saudi Arabia (Research \& Ethics Committee, King Abdulaziz Air Base Hospital; King Abdulaziz Medical City HospitalRiyadh, King Abdullah International Medical Research center IRB; King Abdulaziz University Hospital-Jeddah unit of Biomedical Ethics and Research committee; King Faisal Specialized Hospital \& Research Center - clinical research committee; King Fahd Medical City IRB, King Saud Medical City IRB); Algeria (University Hospital Beni-Messous, Issad Hassani Hospital Ethics Committee); South Africa (Richard van Zyl-Smit Pharma Ethics Ltd, University of Stellenbosch Respiratory Research Unit Health Research Ethics Committee; UCT Lung Institute, Faculty of Health Sciences Human Research Ethics Committee, Charlotte Maxeke Johannesburg Academic Hospital, University of the Witwatersrand Johannesburg Human Research Ethics Committee).

\section{Acknowledgments}

The authors would like to thank all investigators who enrolled patients in this study. To NCT (Cairo, Egypt) for clinical study management, Axonal-Biostatem (Castries, France) for data management and statistics, and Thierry Radeau Consulting (Epinay-Sous-Senart, France) for medical writing support funded by AstraZeneca (United Arab Emirates) in accordance with Good Publication Practice (GPP3) guidelines (https://www.ismpp.org/gpp3).

\section{Disclosure}

NK received a grant from AstraZeneca during the conduct of the study. RVZS received personal fees from AstraZeneca, Novartis, GSK, Chiasi, Cipla, Pfizer, Aspen, Roche and MSD, and grants and personal fees from GSK, outside of the submitted work. ASA received funds from AstraZeneca to conduct the study. WA and EM are employees at AstraZeneca. The authors report no other conflicts of interest in this work.

\section{References}

1. World Health Organization. The top 10 causes of death; 2014 May. Available from: http://www.who.int/mediacentre/factsheets/fs310/en/. Accessed January 30, 2019.

2. Burge S, Wedzicha JA. COPD exacerbations: definitions and classifications. Eur Respir J Suppl. 2003;41:46s-53s. doi:10.1183/090 31936.03.00078002

3. Kessler R, Partridge MR, Miravitlles M, et al. Symptom variability in patients with severe COPD: a pan-European cross-sectional study. Eur Respir J. 2011;37:264-272. doi:10.1183/09031936.00051110

4. Global Initiative for Chronic Obstructive Lung Disease. Global strategy for the diagnosis, management, and prevention of chronic obstructive pulmonary disease (updated 2015). Available from: https:/goldcopd.org/wp-content/uploads/2018/11/GOLD-2019-v1. 7-FINAL-14Nov2018-WMS.pdf. Accessed March 22, 2019.

5. Lopez-Campos JL, Calero C, Quintana-Gallego E. Symptom variability in COPD: a narrative review. Int J Chron Obstruct Pulmon Dis. 2013;8:231-238. doi:10.2147/COPD.S42866

6. van den Berge M, Hop WC, van der Molen T, et al. Prediction and course of symptoms and lung function around an exacerbation in chronic obstructive pulmonary disease. Respir Res. 2012;13:44. doi:10.1186/1465-9921-13-44

7. Partridge M, Karlsson N, Stahl E. Development of the Morning Activities and Symptoms Questionnaire (MASQ) for COPD. Eur Respir J. 2008;32:814s.

8. Partridge MR, Miravitlles M, Ståhl E, et al. Development and validation of the capacity of daily living during the morning questionnaire and the global chest symptoms questionnaire in COPD. Eur Respir J. 2010;36:96-104. doi:10.1183/09031936.00123709

9. Mapel DW, Dalal AA, Johnson PT, et al. Application of the new GOLD COPD staging system to a US primary care cohort, with comparison to physician and patient impressions of severity. Int J Chron Obstruct Pulmon Dis. 2015;10:1477-1486.

10. Safka KA, Wald J, Wang H, et al. GOLD stage and treatment in COPD: a 500 patient point prevalence study. Chronic Obstr Pulm Dis. 2016;4:45-55. doi:10.15326/jcopdf.4.1.2016.0126

11. Decramer M, Celli B, Kesten S, et al. Effect of tiotropium on outcomes in patients with moderate chronic obstructive pulmonary disease (UPLIFT): a prespecified subgroup analysis of a randomised controlled trial. Lancet. 2009;374:1171-1178. doi:10.1016/S01406736(09)61298-8

12. Vestbo J, Anderson W, Coxson HO, et al. Evaluation of COPD longitudinally to identify predictive surrogate end-points (ECLIPSE). Eur Respir J. 2008;31:869-873. doi:10.1183/09031936.00111707

13. Vestbo J, Anderson JA, Calverley PM, et al. Adherence to inhaled therapy, mortality and hospital admission in COPD. Thorax. 2009;64:939-943. doi:10.1136/thx.2009.113662

14. Schauer GL, Wheaton AG, Malarcher AM, et al. Smoking prevalence and cessation characteristics among U.S. adults with and without COPD: findings from the 2011 behavioral risk factor surveillance system. COPD. 2014;11:697-704. doi:10.3109/15412555.2014.898049

15. World Health Organization. Household air pollution and health. Fact sheets (8 May 2018). Available from: https://www.who.int/newsroom/fact-sheets/detail/household-air-pollution-and-health. Accessed January 30, 2019.

16. Kilic H, Kokturk N, Sari G, et al. Do females behave differently in COPD exacerbation? Int J Chron Obstruct Pulmon Dis. 2015; 10:823-830. doi:10.2147/COPD.S78952

17. Greulich T, Weist BJD, Koczulla AR, et al. Prevalence of comorbidities in COPD patients by disease severity in a German population. Respir Med. 2017;132:132-138. doi:10.1016/j.rmed.2017.10.007

18. Miravitlles M, Price D, Rabe KF, et al. Comorbidities of patients in tiotropium clinical trials: comparison with observational studies of patients with chronic obstructive pulmonary disease. Int $J$ Chron Obstruct Pulmon Dis. 2015;10:549-564. doi:10.2147/COPD 
19. Gunen H, Yilmaz M, Aktas O, et al. Categorization of COPD patients in Turkey via GOLD 2013 strategy document: ALPHABET study. Int J Chron Obstruct Pulmon Dis. 2015;10:2485-2494. doi:10.2147/ COPD

20. Miravitlles M, Worth H, Soler Cataluña JJ, et al. Observational study to characterise 24-hour COPD symptoms and their relationship with patient-reported outcomes: results from the ASSESS study. Respir Res. 2014;15:122.

21. Fragoso E, André S, Boleo-Tomé JP, et al. Understanding COPD: a vision on phenotypes, comorbidities and treatment approach. Rev Port Pneumol (2006). 2016;22:101-111. doi:10.1016/j.rppnen.2015. 12.001

22. Arkhipov V, Arkhipova D, Miravitlles M, et al. Characteristics of COPD patients according to GOLD classification and clinical phenotypes in the Russian Federation: the SUPPORT trial. Int J Chron Obstruct Pulmon Dis. 2017;12:3255-3262. doi:10.2147/COPD

23. Di Marco F, Santus P, Scichilone N, et al. Symptom variability and control in COPD: advantages of dual bronchodilation therapy. Respir Med. 2017;125:49-56. doi:10.1016/j.rmed.2017.03.001

24. Wu M, Wang Z, Li M, et al. Daily symptom variability in patients with stable COPD: a narrative review. West $J$ Nurs Res. 2018;40:1543-1561. doi:10.1177/0193945917705132

25. Partridge MR, Karlsson N, Small IR. Patient insight into the impact of chronic obstructive pulmonary disease in the morning: an internet survey. Curr Med Res Opin. 2009;25(8):2043-2048. doi:10.1185/ 03007990903103006
26. Roche N, Chavannes NH, Miravitlles M. COPD symptoms in the morning: impact, evaluation and management. Respir Res. 2013;14:112. doi:10.1186/1465-9921-14-112

27. Kim YJ, Lee BK, Jung CY, et al. Patient's perception of symptoms related to morning activity in chronic obstructive pulmonary disease: the SYMBOL study. Korean J Intern Med. 2012;27:426-435. doi:10.3904/kjim.2012.27.4.426

28. Miravitlles M, Ribera A. Understanding the impact of symptoms on the burden of COPD. Respir Res. 2017;18:67. doi:10.1186/s12931017-0548-3

29. Núñez A, Esquinas C, Barrecheguren M, et al. Evaluating the impact of morning symptoms in COPD using the Capacity of Daily Living during the Morning (CDLM) questionnaire. Int $J$ Chron Obstruct Pulmon Dis. 2018;13:3837-3844. doi:10.2147/COPD

30. Vestbo J, Hurd SS, Agustí AG, et al. Global strategy for the diagnosis, management, and prevention of chronic obstructive pulmonary disease: GOLD executive summary. Am J Respir Crit Care Med. 2013;187:347-365. doi:10.1164/rccm.201204-0596PP

31. van Buul AR, Kasteleyn MJ, Chavannes NH, Taube C. Morning symptoms in COPD: a treatable yet often overlooked factor. Expert Rev Respir Med. 2017;11(4):311-322. doi:10.1080/17476348.2017. 1305894

32. Kokturk N, Polatli M, Oguzulgen IK, et al. Adherence to COPD treatment in Turkey and Saudi Arabia: results of the ADCARE study. Int J Chron Obstruct Pulmon Dis. 2018;27:1377-1388.

\section{Publish your work in this journal}

The International Journal of COPD is an international, peer-reviewed journal of therapeutics and pharmacology focusing on concise rapid reporting of clinical studies and reviews in COPD. Special focus is given to the pathophysiological processes underlying the disease, intervention programs, patient focused education, and self management protocols. This journal is indexed on PubMed Central, MedLine and CAS. The manuscript management system is completely online and includes a very quick and fair peer-review system, which is all easy to use. Visit http://www.dovepress.com/testimonials.php to read real quotes from published authors. 